\title{
Les espaces de la violence
}

\author{
Une étude de cas sur le meurtre des Juifs en Ukraine
}

(1941-1944)

\author{
Michaela Christ \\ (Docteur en sociologie à l'Université de Flensburg) \\ Traduction : Daniel Bonnard
}

\section{Résumé/Abstract}

[Fr] En se penchant sur l'occupation de la ville ukrainienne de Berditchev et le meurtre de 18.000 habitants juifs par des SS et des policiers allemands, cet article examine les transformations de l'espace au travers de la violence de masse autant en termes sociaux que topographiques. Immédiatement après avoir pris la ville, les Allemands commencent à utiliser l'espace en le restructurant et en le modelant en fonction de leurs intérêts. Le parc du centre-ville se mue en cimetière pour les soldats allemands tombés durant la prise de la ville. Les écoles servent de zone d'accueil pour les troupes de la Wehrmacht, tandis que les habitants doivent quitter leur maison pour laisser place aux militaires et au personnel civil allemand. Tous les Juifs sont forcés de se déplacer dans les quartiers les plus pauvres et les plus sales, avant d'être rassemblés, assassinés et enterrés dans le voisinage immédiat de la ville. Dans l'étude de ces pratiques d'occupation et d'annihilation, cet article s'interroge en même temps sur la manière dont les Juifs et les Ukrainiens y réagissent. Il montre comment la ville de Berditchev est devenue la ville allemande de Berditschew, en soutenant que les transformations spatiales dans leur dimension sociale et topographique ont été parallèlement produites par tous les groupes, et en quoi ils ont été l'objet de nouvelles assignations et appropriations par tous.

Mots-Clés : Violence, espace, ordre social, Shoah, Ukraine, analyse de la pratique.

[En] Examining the occupation of the Ukrainian city of Berdichev and the murder of some 18,000 Jewish inhabitants by German SS and police, this paper explores the transformation of space in both social and topographical terms by means of mass violence. Immediately after they had seized the town, the Germans started to use the location for their interests, thus restructuring and remodeling it. For example, the park in the city center was transformed into a cemetery for German soldiers killed in action during the conquest, schools gave shelter to Wehrmacht troops, townspeople had to leave their houses for German military and civil personnel, and all Jews were forced to move to the poorest and dirtiest neighborhood before they were rounded up, shot, and buried in the immediate vicinity of the town. This paper focuses on 
these practices of occupation and annihilation and asks how Jews and Ukrainians reacted to them, thus taking all agents into account. It shows how the city of Berdichev turned step by step to the German city of Berditschew, arguing that social and topographical spaces were created separately by all groups and at the same time all groups participated in mutual naming and appropriation.

Keywords: Violence, space, social order, Holocaust, Ukraine, praxeology

\section{Introduction}

Spatialité et violence sont des catégories étroitement liées. D'abord, et de manière évidente, parce que la violence est toujours le fait d'un lieu. Elle est vécue et exercée en des lieux concrets. C'est le premier type de rapport qu'entretient la violence avec l'espace, en ce qu'il n'est guère possible de traiter d'une pratique de violence sans prendre en compte ses effets dans l'espace. Le deuxième type de rapport entre spatialité et violence découle du fait qu'à l'inverse, lorsqu'il est question d'un lieu, il est toujours question de sa spatialité dans le cadre de rapports sociaux, qu'il s'agisse d'un pays, d'une région, d'une ville, d'une rue, d'une maison ou d'une chambre. En faisant, par exemple, référence à Istanbul dans une conversation, nous signifions à nos interlocuteurs que nous parlons de la ville d'aujourd'hui, ou au contraire que nous évoquons les temps anciens en utilisant alors le nom de Constantinople ou de Byzance. Dès lors que nous parlons d'un lieu, nous le faisons toujours en mobilisant les dimensions spécifiques temporelles, sociales et culturelles constitutives de celuici (Löw, 2001). Cette caractéristique ne s'applique pas qu'à l'espace. La violence et l'exercice de la violence sont également construits socialement, culturellement et au niveau temporel. Ce sont des espaces et des contextes sociaux, temporels et culturels donnés qui déterminent qui peut ou doit exercer quel type de violence en quel lieu et envers qui (Christ, 2013). Enfin, et cela constitue le troisième point que je souhaite relever, le rapport qu'entretiennent violence et spatialité est de caractère relationnel. La violence s'inscrit dans l'espace. C'est ainsi que tout lieu voit sa topographie marquée par les formes de violence collective perpétrées, comme tout espace de relations sociales se voit transformé par les violences. Inversement, dans certaines configurations données sont produites certaines violences extrêmes ou, du moins, l'espace social vient influencer le type de pratique violente mis en œuvre. La spatialité donne forme à la violence.

Dans le cadre de cette contribution, je vais utiliser un exemple afin d'illustrer de manière concrète la relation entre violence et spatialité. Il sera question de la ville de Berditchev ${ }^{1}$ durant la Seconde Guerre mondiale (pour une présentation détaillée, voir Christ, 2011). Avant la guerre, les Juifs représentaient près de la moitié des

\footnotetext{
${ }^{1}$ Il s'agit de la traduction française de l'ukrainien Бердичів ou du russe Бердичев.
} 
60.000 habitants de cette ville moyenne de l'ouest de l'Ukraine. Les traces de la vie juive à Berditchev remontent au $\mathrm{XVI}^{\mathrm{e}}$ siècle. Durant les siècles suivants, la ville devient un centre important de la culture juive, autant sur le plan intellectuel que religieux. Encore aujourd'hui, un pèlerinage a lieu sur la tombe du Rabin Levi Yitzchaks, un érudit du judaïsme hassidique, enterré en 1809 à Berditchev². Sous la rubrique «Russie», les revues et journaux juifs de langue allemande rendent compte, dès la moitié du XIX ${ }^{\mathrm{e}}$ siècle, des nouvelles de Berditchev et parlent de la ville abritant plus de cinquante synagogues comme du «Moscou juif ${ }^{3}$. Aux côtés d'Odessa, de Kiev et de Lviv (anciennement Lvov), Berditchev est, à cette époque, une des plus grandes villes juives d'Ukraine, les Juifs y représentant plus de 90 pourcent des 500.000 habitant-e-s ${ }^{4}$. Au début du $\mathrm{XX}^{\mathrm{e}}$ siècle, la ville compte un tribunal juif, des dizaines de synagogues et de lieux de prière, ainsi qu'un théâtre juif dans lequel des pièces de troupes de théâtre réputées sont régulièrement à l'affiche. Des revues et des livres imprimés ou édités à Berditchev en hébreu, en yiddish et en russe avant la Première Guerre mondiale figurent toujours à l'inventaire de nombreuses bibliothèques aux USA, en Europe et en Israël. Sur d'anciennes cartes postales, des photos des rues de la ville montrent de nombreuses enseignes de magasins en yiddish ou en hébreu. Jusqu'à la fin des années trente, des écoles juives, russes et ukrainiennes fonctionnent en parallèle. Pendant les deux premières décades du régime soviétique et malgré les difficultés considérables liées aux nouvelles formes d'acculturation et de sécularisation, une vie culturelle juive se maintient, et ce jusqu'aux «purges staliniennes » de 1937/1938. Celles-ci voient alors de nombreux Juifs perdre leurs emplois dans la fonction publique, tandis que les écoles juives, les associations culturelles, les maisons d'édition et de nombreuses institutions sont contraintes de fermer ${ }^{5}$.

Au début du mois de juillet 1941, les Allemands occupent la ville. Dès lors, la ville se transforme rapidement. Ces changements sont entraînés par la violence, le meurtre, le pillage, la violence sexuelle, le travail forcé, l'humiliation et l'exploitation. La domination allemande dure deux ans et demi. En l'espace de seu-

\footnotetext{
${ }^{2}$ À propos de Levi Yitzchaks, cf. Petrovsky-Shtern, Y. (2004), “The Drama of Berdichev : Levi Yitshak and his Town ", Polin, 17, pp.83-95.

${ }^{3}$ Allgemeine Zeitung des Judenthums du 29 avril 1844, p.246.

${ }^{4}$ Encyclopedia of Ukraine, Kubijovyc, V. (dir), Toronto/Buffalo/London 1984-1993, p.204.

${ }^{5}$ Les dites « grandes purges » staliniennes ne sont pas dirigées explicitement contre la population juive, bien que de nombreux Juifs, faisant partie des couches urbaines et qualifiées et ayant fait carrière dans le parti, l'administration, la science, la culture, l'armée ou la police, se trouvent persécutés en tant qu' «ennemis du peuple», cf. Snyder, T. (2008), The Life an Death of Western Volhynian Jewry, 1921-1945, p.88, in Brandon, R., Lower, W. (Hg.), The Shoah in Ukraine. History, Testimony, Memorialization, Bloomington/Indianapolis, pp.77-113. Sur la survivance de la culture juive, cf. Veidlinger, J (1998). Let's Perfom a Miracle: The Soviet Yiddish State Theater in the 1920s, Slavic Review, 57/2, pp.372-397.
} 
lement trente mois, Berditchev est changée de fond en comble. La ville russoukrainienne de Berditchev, empreinte de culture juive, devient en un laps de temps restreint un lieu sous domination allemande du nom de Berditschew. Cette ville, sans avoir connu de modification profonde de son apparence suite à la guerre, fonctionne selon des règles radicalement différentes et voit la structure de sa population bouleversée. Car, trois mois seulement après le début de l'occupation, il ne reste plus à Berditchev qu'une petite centaine des 30.000 habitant-e-s juifs de Berditchev que comptait la ville. Environ 18.000 Juifs, principalement des femmes, des enfants et des personnes âgées, ont été abattus par des membres des Einsatzgruppen et des bataillons de police durant les premières semaines successives à la prise de la ville. Cela correspond environ à un tiers de la population totale. Le reste des Juifs de Berditchev a réussi à quitter la ville avant l'arrivée des Allemands.

Les événements qui ont concerné la ville de Berditchev/Berditschew ne sont pas un cas isolé. Quel que soit le lieu, la façon dont les puissances occupantes ont procédé a été sensiblement identique. Les dynamiques sociales qui font l'objet de cette contribution, pourraient être décrites - mises à part quelques spécificités - pour des centaines d'autres villes et villages dans les territoires soviétiques occupés par les Allemands. De manière plus générale, on retrouve les traits fondamentaux de ce rapport entre violence et espace dans d'autres situations relevant des violences de masse asymétriques contre des populations. Les similitudes constatées ne doivent toutefois pas faire oublier les différences, qui sont significatives. Plus l'armée allemande progresse à l'Est, plus le nombre de Juifs survivant aux massacres se trouve élevé dans les localités assiégées. L'explication réside dans le fait que plus l'invasion dure, plus cela laisse du temps pour fuir. Or, il ne s'écoule que quinze jours entre le déclenchement de l'invasion de l'URSS le 22 juin 1941, dite «Opération Barbarossa », et l'arrivée de l'armée allemande à Berditchev. Il est clair que le laps de temps à disposition pour prendre des décisions impératives, qui relèvent souvent de l'existentiel - fuir ou rester, participer à l'évacuation ou non - est très restreint. La progression rapide de la Wehrmacht sur le front ne laisse guère aux habitant-e-s des régions limitrophes de la frontière le temps de réfléchir à une échappatoire ou même d'envisager des préparatifs pour fuir leurs lieux de résidence. Au niveau des villes occupées par les troupes allemandes durant les quatre premières semaines de l'invasion de l'URSS, Mordechai Altshuler estime à deux tiers la proportion de la population juive habitant à l'origine dans les zones envahies qui est sujette à l'occupation. Seul un tiers réussit donc à quitter la région à temps (Altshuler, 1993, 97). 
Cette étude de cas se base sur des documents d'archives de la période étudiée, comme les fonds des Gebietskommissariat ${ }^{6}$ de « Berditschew » et de «Schitomir ». À cela s'ajoute l'analyse des témoignages de survivants juifs et non-juifs, principalement issus du fonds de la Shoah Foundation, ont été d'un apport considérable. Ce corpus a été complété par la lecture de documents provenant de procès allemands conduits dans l'après-guerre, ainsi que d'un nombre important de diverses autres sources.

Au préalable, il convient de clarifier une question liée à l'emploi des noms de lieux. Il sera ici volontairement fait usage à la fois du nom russe et du nom allemand, afin de souligner que les noms de lieux expriment bien d'avantage que le simple habitat humain qu'ils désignent. Les noms de lieux sont l'expression d'un espace dans son inscription sociale donnée et spécifique. L'historien Karl Schlögel fait systématiquement référence à cette dimension dans ses travaux (Schlögel, 2003). Ainsi, les noms de lieux portent la marque de l'histoire impériale ou coloniale. Ils peuvent signaler des moments de rupture dans la continuité des formes de domination, ils témoignent des hiérarchies et de la distribution des rapports de pouvoir. La façon dont les lieux sont nommés nous donne des indications sur les différentes formations sociales, sur les systèmes juridiques et sur les espaces dans lesquels s'exerce la domination. En raison des rapports de domination en vigueur à l'époque étudiée, durant laquelle l'Ukraine fait partie de l'Union soviétique, Berditchev est le nom russe de la ville.

Traiter d'espaces particuliers signifie aussi traiter d'espaces qui sont le fait d'appropriations sociales par des personnes données, à une époque donnée. Par làmême, je situe les événements décrits à Berditschew dans un cadre plus général constitué par les expériences de générations d'hommes, de femmes et d'enfants vivants ensemble, marqués et socialisés dans et par la guerre à l'Est. J'y comprends tout autant les Ukrainiens et les Allemands, les Juifs comme les non-Juifs.

\section{Avant l'occupation : la présence des réfugiés}

Revenons-en à Berditchev. La transformation de l'espace urbain en Berditschew ne commence pas à proprement parler au moment où les soldats de la Wehrmacht sillonnent les rues de la ville, juchés sur leurs motos, annonçant la supériorité de l'armée allemande. Berditchev a déjà commencé à se transformer alors que le front se situait quelques centaines de kilomètres plus à l'Ouest. Des centaines, voir des

\footnotetext{
${ }^{6}$ Les Gebietskommissariat sont les subdivisions administratives introduites, au niveau municipal, par les Allemands dans les territoires occupés en Europe de l'Est et placés sous l'autorité du Reichskommissar Ostland et du Reichskommissar Ukraine, eux-mêmes subordonnés au Reichsministerium für die besetzten Ostgebiete (Archives fédérales allemandes, cote R6, inventaire en ligne), NdT.
} 
milliers de réfugiés arrivent alors dans la ville. Un axe ferroviaire important vers l'Est, ainsi que deux grandes voies de transit vers Kiev, font de la ville une étape importante pour de nombreuses personnes en fuite vers d'autres régions situées plus à l'intérieur du pays. Ce sont avant tout des Juifs qui fuient la violence exercée par les troupes allemandes, souvent déclenchée dès leur arrivée dans les villes assiégées.

Les réfugiés occupent une place particulièrement défavorisée dans la hiérarchie sociale de la ville, ce qui influence la dynamique des violences : ils apportent des informations à Berditchev, ils sont porteurs de récits sur les Allemands, sur les crimes commis contre les Juifs et rendent clairement compte de la menace mortelle que peut faire peser l'occupation allemande. Or, les réfugiés ne font pas que colporter vers Berditchev d'horrifiantes nouvelles sur la politique des Allemands. Leur présence en tant que telle revêt une signification. Les réfugiés sont un indicateur de la façon dont la configuration sociale se modifie en un lieu donné, dès que les Allemands sont installés au pouvoir.

La présence des réfugiés rend tangible le fait que les Juifs courent un grand danger. En outre, leur situation à Berditchev devient fragile et précaire avant même que l'occupation n'ait commencé. Durant lesdits «jours de l'anarchie »-ce terme est utilisé dans les témoignages des survivants pour désigner le laps de temps séparant le retrait de l'Armée rouge de l'arrivée de la Wehrmacht - cette précarité prend un caractère particulièrement prononcé.

En effet, plus le front s'approche de la ville, plus le fonctionnement de celle-ci revêt un caractère chaotique. Au fait que plusieurs milliers de civils - Juifs pour la plupart - quittent la ville, s'ajoute l'évacuation plus ou moins organisée, menée par les autorités soviétiques. Tandis que certains Juifs y prennent part, la majorité de ceux qui se sont enfuis le font de leur propre initiative. Les équipements industriels sont démontés pour être mis à l'abri des Allemands. Des parcs de machines entiers et du matériel sont chargés sur des trains de marchandises, à la suite desquels de nombreux travailleurs et travailleuses fuient vers l'Est. De la même manière, les employés de l'administration municipale ainsi que les soldats de l'Armée rouge prennent la fuite. Nahum Epelfeld, âgé de 13 ans à l'époque, se souvient : «Le 5 juillet 1941, Berditchev était méconnaissable. Les rues étaient remplies de troupes en train de battre en retraite. Des colonnes de soldats épuisés défilaient le long des rues et on voyait le désespoir, la consternation sur les visages. Les soldats s'étaient changés en une masse de réfugiés » (Epelfeld, 2004, 110).

Le retrait de l'Armée rouge et de l'administration soviétique, désormais incapable de défendre efficacement la ville et d'y maintenir l'ordre, provoque dès lors une sorte de désécurisation de l'espace. Une fois l'évacuation effectuée, il n'y a soudain plus d'instance à même de régler les conflits d'intérêts au sein de la population. De même, il n'existe plus d'instance susceptible de représenter les intérêts de la population auprès des Allemands. Le retrait aux allures de panique des autorités sovié- 
tiques indique aux habitant-e-s de Berditchev restés dans la ville que leur sécurité, leur intégrité corporelle et la protection de leur propriété ne sont plus garanties.

Ce sont, dans leur grande majorité, des femmes, des enfants et des personnes âgées qui restent dans la ville. De nombreux bâtiments, appartements, commerces et installations industrielles sont laissés à l'abandon. Les incertitudes du futur, la menace d'une occupation imminente et les opportunités offertes par les maisons vides en nombre infini sont autant de facteurs qui conduisent les habitant-e-s demeurés sur place à commettre des pillages. Les appartements des Juifs, en premier lieu, sont dévalisés. Comme les Juifs ont représenté la majorité des personnes à prendre la fuite, leurs maisons sont pour la plupart vides et sans surveillance. Nahum Epelfeld, adolescent à l'époque, décrit dans ses mémoires une période marquée par l'absence de règles et de lois : «Le 7 juillet, des délits tels que le rapt ou les coups et blessures étaient monnaie courante en ville : on défonçait les portes, faisait sauter les cadenas, on emportait tout ce qui n'était pas scellé ou fixé au sol » (Epelfeld, 2004, 111).

L'absence d'institution de maintien de l'ordre, pendant plusieurs jours, pousse aussi certains à s'enrichir là où les propriétaires sont encore présents. Maria Beizerman fait le récit d'une situation relative à ce moment qui précède l'occupation, durant laquelle un homme essaye de voler les pigeons de l'élevage de son frère :

«Mon frère élevait des pigeons et notre voisin élevait aussi des pigeons. Avant l'arrivée des Allemands, les gens ont simplement mis la main sur un couple de pigeons de mon frère. Et le voisin voulait aussi des pigeons. Alors, mon père est sorti de la maison, est allé dans la cour et l'a menacé. Ce sur quoi, le voisin lui a répondu :'Toi, le Juif, tu me le payeras! Ainsi, lorsque les Allemands se sont mis à tuer, il a dénoncé mon père et mon frère. » (Interview de Beizerman)

C'est pourquoi on constate, avant l'arrivée de la Wehrmacht, un déplacement des rapports de pouvoir dans le lieu étudié, en défaveur de la population juive. L'espace social se transforme profondément et à une cadence avancée. On observe des changements qui portent sur plusieurs plans. Les règles déterminant qui est autorisé à interagir avec qui, et quelles sont les hiérarchies en vigueur dans ce type de relations, ne résultent pas de processus sociaux et historiques de longue durée, mais dépendent de l'occupant. Dans ce sens, la partie suivante de cet article sera consacrée à l'analyse des hiérarchies sociales à l'œuvre dans la ville.

\section{De Berditchev à Berditschew}

Une des premières mesures prises au commencement de l'occupation consiste en une sorte d'inventaire des habitant-e-s de Berditschew. L'administration militaire allemande ordonne aux habitant-e-s juifs de se faire recenser par les autorités. Le recensement a surtout pour fonction d'établir rapidement le nombre et le lieu de résidence des habitant-e-s juifs. De manière concomitante au recensement, il est donné l'ordre de porter la dite «étoile juive ». Tous ceux qui se font enregistrer sont obli- 
gés de coudre une étoile jaune, à six branches, de façon visible sur leurs vêtements. Le fait de ne pas respecter cet ordre est sévèrement puni, comme s'en souvient Mikhail Aranowitsch Vanshelboim : "Dans le décret, il était aussi écrit que si l'on était attrapé sans étoile, on était fusillé sur-le-champ. Ce décret a été affiché partout en ville » (Interview de Vanshelboim).

Dès à présent, les Juifs sont reconnaissables au premier coup d'œil, il est aisé de contrôler s'ils respectent le large éventail de nouvelles règles édictées par l'administration. Les Juifs ne sont pas autorisés à faire leurs courses au marché, des règles spéciales de sortie leur sont appliquées le soir, ils n'ont pas le droit de prendre le tram, et ainsi de suite. Par conséquent, il devient difficile aux Juifs d'organiser leur quotidien.

Le port obligatoire de l'étoile divise la population de Berditschew en imposant de nouvelles hiérarchies sociales. D'un côté, les Juifs dont l'existence est drastiquement restreinte, de l'autre les habitant-e-s non-Juifs de Berditschew, dont la vie connaît certes des changements, mais qui peuvent espérer s'arranger avec la nouvelle situation, du moins au début de l'occupation. Les personnes rattachées à la puissance d'occupation forment un troisième groupe, dont les possibilités - que ce soit en termes d'action ou de comportement - dépassent de beaucoup celles des autres habitants. Du fait de cette division, les Juifs occupent la place la plus basse de la hiérarchie sociale. Leur vie se trouve réglementée par le port de l'étoile, ils sont rendus visibles en tant que Juifs, c'est-à-dire livrés aux persécutions mises en œuvre par l'occupant. En tant que technique de contrôle, la perfidie de l'étoile réside dans le fait qu'elle transforme les habitant-e-s non-juifs de Berditschew en surveillants officieux des Juifs. Celui qui, indépendamment du motif, ne se préoccupe pas de savoir si son voisin, son collègue de travail ou ami juif suit les instructions des Allemands, court le risque d'être lui-même soupçonné. Et, dans ce cas, le soupçon porte sur l'aide aux Juifs. Car on peut partir du principe que les habitant-e-s non-juifs de Berditschew se sentent eux aussi observés et contrôlés. Pour eux aussi, de nouvelles règles entrent en vigueur. Eux aussi sont contraints de s'adapter à la nouvelle situation, de se l'approprier et de ne pas entrer en conflit avec l'occupant, délibérément prêt à faire usage de la violence à tout moment.

Le port obligatoire de l'étoile jaune divise également la ville au niveau topographique. Car, avec cette technique de contrôle, le monde de Berditschew se voit divisé définitivement en certains lieux où les Juifs ont l'autorisation d'entrer, et en d'autres lieux dont l'accès ne leur est plus permis. La plupart des habitant-e-s juifs de Berditschew essaient, dès le début de l'occupation, de sortir le moins possible de leur maison. Ils tentent d'éviter les lieux publics, afin de se protéger des agressions ou bien pour éviter des rencontres désagréables avec les habitant-e-s non-juifs. Par l'intermédiaire du port obligatoire de l'étoile jaune s'établit l'interdiction systématique faite aux Juifs d'utiliser librement et sans restriction l'espace urbain. Le port obligatoire de l'étoile est ainsi une mesure qui, loin de relever seulement de la So- 
zialpolitik $^{7}$, donne un moyen de réorganiser Berditschew en termes d'espaces sociaux et de topographie. Avant même que le ghetto ne soit érigé et que les Juifs y soient enfermés comme dans une prison, il existe en ville des lieux qui leur sont interdits à cause du port obligatoire de l'étoile jaune.

Venons-en maintenant aux changements matériels survenus dans le paysage urbain. Il est important ici d'évoquer un aspect marquant : durant quasiment toute la durée de l'occupation, Berditschew est pour ainsi dire peuplée de soldats. Comme il s'agit d'un carrefour important, la ville fait office de plaque tournante pour le transport des personnes et des marchandises, aussi bien sur l'axe Est-Ouest que NordSud. Des dizaines de milliers de soldats font étape à Berditschew pendant la guerre, nombre d'entre eux y passent des jours, voire des semaines, en attendant d'être transportés en d'autres lieux. Comme la guerre implique le déplacement de gigantesques quantités de personnes, les administrations militaires et civiles des territoires soviétiques occupés par les Allemands sont confrontées à des défis logistiques considérables. L'occupation nécessite donc d'abord de la place.

À Berditschew, les bâtiments les plus prestigieux, les plus spacieux et les moins éprouvés par les combats sont réquisitionnés et utilisés par l'administration militaire. Il en va de même des installations industrielles. Les écoles sont fermées. Elles servent désormais d'hébergement aux soldats de la Wehrmacht. Une fabrique de savon est transformée en dépôt de munitions, un moulin en entrepôt à provisions. Des maisons sont confisquées et aménagées pour y établir l'administration militaire, les quartiers des officiers, un casino, un hôpital militaire, un bordel et pour d'autres affectations. En termes de travaux de réparation et d'entretien, les Allemands ont recours à des prisonniers de guerre russes et à la population locale. Toutes ces mesures propres aux nécessités matérielles de l'occupation changent le visage de la ville. Je vais, dans les paragraphes suivants, développer un exemple d'appropriation de l'espace urbain - en l'occurrence un cimetière -, afin de montrer en détails comment s'opère la relation entre l'accaparation violente du terrain et la transformation subséquente de l'espace social.

L'enterrement des soldats allemands tombés durant la prise de la ville au centre de l'agglomération est une des premières mesures à modifier le paysage urbain. Un parc au cœur de Berditschew devient ainsi un cimetière militaire allemand. Le fait d'enterrer des soldats en pleine ville n'est pas inhabituel en période de guerre. La nécessité de mettre les morts rapidement en terre à cause de la chaleur estivale et de la progression du front mène à la transformation des parcs urbains en cimetières dans de nombreuses villes des territoires occupés. Toutefois, les pratiques sociales sont des formes de communication et l'installation d'un cimetière pour des soldats

\footnotetext{
${ }^{7}$ Le terme de Sozialpolitik désigne un ensemble de décisions politiques visant à changer la situation économique et sociale de groupes sociaux, les mesures appliquées pouvant être à l'avantage ou au désavantage de ces derniers, NdT.
} 
allemands est, dans ce sens, un acte particulièrement éloquent. Car ce même parc, situé au cœur de la ville, possède une haute valeur symbolique. Le fait d'y enterrer les soldats tombés au front représente un geste d'autorité, qui veut démontrer de manière concrète et dans un lieu connu qui a gagné la bataille pour la ville et à quel prix. Le cimetière militaire exprime le message suivant : le salaire obtenu en retour des peines concédées consiste dans le pouvoir d'aménager le lieu conquis selon ses propres besoins. Le choix du lieu revêt une signification supplémentaire dans le sens où un ancien cimetière juif, qui n'est plus utilisé, occupe une partie du parc. Les tombes juives du parc sont détruites pendant l'occupation et probablement utilisées pour des travaux de construction dans la ville.

Dans la perspective des Allemands, la mise en terre de leurs morts en un lieu qu'ils veulent dédier à la commémoration représente une forme de sédentarisation. Le cimetière se trouve au sommet de la ville, au croisement de deux voies importantes de transit, incontournables pour qui veut traverser l'agglomération. Ce sont environ 200 tombes qui, entretenues avec attention (Volksbund Deutsche Kriegsgräberfürsorge, G.N. 1.2. Lr), signifient à la population locale que les nouveaux maîtres de la ville se préparent à un séjour de longue durée.

Les bouleversements politiques, avec leurs conséquences sur l'organisation de la société, sont presque toujours accompagnés de l'imposition de nouveaux noms de rues et de places. Le conquérant s'arroge ainsi le droit de donner un nom à ce qu'il a accaparé. Dans les territoires occupés d'Ukraine, les instructions données précisent : «Les noms de rues et de places qui rappellent, de quelque manière que ce soit, le bolchévisme ou des personnes bolchéviques, sont à remplacer» (USHMM $1996 \mathrm{~A}$ 0269).

À Berditschew, de nombreux noms de rues en vigueur jusqu'alors sont traduits en allemand, tandis que d'autres rues reçoivent des noms complètement nouveaux; ainsi, la rue Adolf-Hitler ne manque pas au tableau. Les anciens noms sont supprimés, pendant que les nouveaux noms sont inscrits sur des cartes qui servent à l'orientation des troupes d'occupation. L'acte, apparemment accessoire, consistant à changer les noms des rues contribue ainsi à changer de manière non négligeable la perception du lieu occupé. Ainsi, la Ortskommandantur de Berditschew se trouve à la Bahnhofstrasse et non à la Danilowskaja, on circule sur la Waldemarstrasse au lieu de la Nikolskaja ou bien on emprunte la Brückenstrasse qui mène à la caserne des blindés. De telles appellations sont autant d'occasions offertes aux membres de la puissance occupante de lire les nouveaux noms, de les prononcer à voix haute, de leur donner un sens, ce qui signifie aussi rencontrer des expressions familières et donc considérer, peu à peu, les lieux comme leurs. Le changement des noms des rues, des places et des bâtiments est un acte symbolique, qui, loin de se contenter de rebaptiser certains emplacements, fait émerger de nouveaux espaces sociaux.

Toute société use des noms de rues comme autant de signes visant à exprimer la reconnaissance envers telle personne ou tel événement. Les noms de rues font donc 
également office de monuments commémoratifs. Ils ont, de par cette qualité, une signification beaucoup plus importante que les monuments bâtis, dont l'architecture perd de sa force mémorielle avec le temps, alors que les noms de rues perdurent dans les mémoires et les bouches, du moins celles des riverains. Les noms de rues ne sont pas seulement lus comme des indications servant à l'orientation, mais également comme des signes des rapports de domination (Maurer, 2008). Ils symbolisent le système de valeurs de celui qui les attribue. À Berditschew, les nombreux plans de ville dessinés par l'occupant constituent des documents attestant la soumission de l'espace conquis. Il est frappant de constater que ces plans, porteurs d'une toponymie nouvelle, ne donnent que très peu d'indications sur la vie avant l'occupation allemande.

Avec les troupes allemandes, la violence, la cruauté et l'arbitraire font leur entrée à Berditschew. Ce sont en premier lieu les Juifs, qui, dès les premiers jours de l'occupation, sont humiliés, maltraités, tabassés publiquement, objets de moqueries, victimes de mutilations et de violence sexuelle. Le nouvel agencement de l'espace social rend possible cette violence et en crée les opportunités.

La violence massive à l'encontre des Juifs n'est pas sans provoquer des réactions de la part de la population non-juive. Celle-ci essaie, dans la mesure du possible, d'échapper à la violence. Afin de se protéger, ainsi que de protéger ses biens des agressions des Allemands, les habitant-e-s non-juifs marquent leurs maisons. Ils clouent des croix en bois sur leurs portes et posent des icônes à leurs fenêtres. L'utilisation de symboles chrétiens orthodoxes signale que des chrétiens, et non des Juifs, habitent dans les maisons marquées. Maria Beizerman, une survivante, décrit ceci de la manière suivante : «Là où les Russes vivaient, c'était encore avant les exécutions, avant le pogrom, partout où les Russes vivaient, il y avait des croix accrochées aux portes. Ils ont confectionné des croix à base de petites planches et les ont accrochées au mur. Pour ne pas qu'ils soient confondus par hasard. Pour ne pas que les Russes soient pris à la place des Juifs. Ils savaient bien que là où il y avait une croix, habitaient des Russes, là où il n'y avait pas de croix, habitaient des Juifs » (Interview Beizerman). La volonté de protéger sa propre vie, sa famille, ses biens est tout à fait compréhensible au regard des conditions. Pourtant, ce genre d'attitude a des conséquences qui vont bien plus loin que la fonction de protection originellement visée. Car les croix contribuent à rendre les habitations juives plus faciles à identifier. Ces pratiques des Ukrainiens s'inscrivent ainsi dans celles des Allemands visant à ostraciser les Juifs de ce qu'ils considèrent comme une communauté basée sur l'appartenance.

Concernant, l'utilisation des croix et des icônes, il est intéressant de constater qu'il ne s'agit pas d'une invention datant de l'occupation. Ces pratiques font, au contraire, référence aux expériences effectuées par les populations avec la violence anti-juive. Dans la littérature, on trouve diverses indications sur l'utilisation des croix et des icônes comme mesures de protection pendant les pogroms contre les juifs au XIX 
siècle. Là aussi, les croix devaient empêcher les auteurs de violence de s'attaquer aux maisons où ne résidaient pas de Juifs (Weinberg, $1998 ;$ Klier, 1992). Cet aspect montre en quoi il est nécessaire de prendre en considération le savoir transmis sur la violence et les expériences de violence dans l'analyse des pratiques de violence, en plus de son caractère situé.

À peine deux mois après le début de l'occupation, les Allemands érigent une sorte de ghetto, dans le quartier le plus pauvre et le moins apte à abriter des habitations de toute la ville. Tous les Juifs de Berditschew doivent y loger durant trois semaines, alors que la place est très restreinte. Située directement sur la rive du fleuve, cette partie de la ville est toujours humide, le sol marécageux, la substance des bâtisses détériorée, les maisons petites, miséreuses et délabrées. Tandis que l'occupant s'installe au centre de Berditschew et y accapare les places et bâtiments les moins détruits et les plus prestigieux, le lieu vers lequel on bannit les Juifs est le plus miteux. L'espace situé entre ces deux extrémités, une sorte d'interstice dans la hiérarchie sociale et territoriale, est laissé à la population locale non-juive. De cette façon, les différences entre les vainqueurs et les vaincus, pour être plus précis entre Allemands, Juifs et Ukrainiens, deviennent visibles et sont reproduites par l'agencement de l'espace. Le contraste toujours plus fort entre des habitant-e-s juifs du ghetto poussés à la misère d'un côté, et les membres de la puissance occupante avec une meilleure situation de l'autre, fournit une légitimation au sentiment de supériorité éprouvé par les Allemands. On assiste dès lors à la production d'un ordre spatial, à la fois défini par les Allemands selon des rapports de supériorité imaginaires et rendu concret par la position des personnes dans l'espace, une fois les habitant-e-s juifs déplacés dans le ghetto. Sous l'effet de l'exclusion sociale, se déploie un espace dont la topographie est clairement délimitée et dans lequel la position des Juifs dans la hiérarchie sociale de la ville est ramenée à leurs nouvelles conditions d'existence. Ils ont été, par la violence, réduits à l'état de marginaux (Außenseiter) : en termes sociaux, comme au regard de leur relégation à la marge de l'agglomération.

Le point final de ce processus - du moins dans la perspective des Juifs de Berditschew - consiste en l'assassinat de la grande majorité des habitant-e-s juifs demeurés dans la ville, le 15 septembre 1941. Ce jour-là, des policiers allemands et les membres d'un Einsatzgruppe, aidés de policiers ukrainiens, abattent plus de 12.000 Juifs. Ils sont conduits en plein jour hors du ghetto vers un champ dans les environs immédiats de la ville. Ceux qui ne peuvent pas encore ou ne peuvent plus marcher sont chargés sur des camions et conduits au lieu d'exécution. En cet endroit, des prisonniers de guerre russes ont dû excaver cinq grandes fosses durant les jours précédents. Les exécuteurs (Täter) forcent les personnes qui sont à leur merci à se dévêtir et à se séparer de leurs objets de valeur. Par groupes de vingt, les personnes doivent se tenir au bord de la fosse, sont abattues et tombent à l'intérieur. Tous les exécutés ne meurent pas immédiatement et de nombreux blessés tombent dans la fosse. Les suivants les recouvrent de leurs corps. Les fosses, contenant encore les vivants et les 
morts, sont recouvertes de terre par des prisonniers de guerre et des habitant-e-s, dans le cadre de travaux forcés.

Une fois la tuerie terminée, une fois les fosses comblées, plus rien ne rappelle ceux qui y ont péri et plus rien ne rappelle les crimes. Ces champs et ces prairies n'ont pas de tombes, il n'y a ni cimetière, ni lieu dont quiconque pourrait chercher la trace. La façon dont les morts sont traités est révélatrice de la valeur qu'attribuent les Allemands aux habitant-e-s juifs de Berditschew. Ils ont été enfouis comme des déchets, sans qu'aucun lieu ne permette leur commémoration.

\section{Conclusion}

La violence est une forme d'agir social (soziales Handeln), elle participe à la définition de l'espace dans lequel les êtres humains interagissent. À Berditschew, l'espace fait l'objet, dès le début de l'occupation, de marquage, de destruction, de nouvelle dénomination et de remodelage. Cela vaut autant pour l'espace compris dans sa dimension sociale que pour la topographie de la ville. Les Allemands établissent, au travers de leurs pratiques, un cadre contraignant qui fixe à la fois ce qui est possible et ce qui ne l'est pas, mais aussi qui est autorisé à faire quoi et de quelle manière. Ils posent les jalons d'un cadre de référence (Erwin Goffman) qui détermine ensuite la vie des habitant-e-s de la ville.

De façon caractéristique, la production et la destruction d'espaces sociaux et topographiques sont le fait de l'interaction entre tous les habitant-e-s de la ville. Tous les habitant-e-s de Berditschew, Juifs, non-Juifs ou Allemands, participent de manière mutuelle, chacun à sa façon, et au regard des rapports de pouvoir extrêmement inégaux, de manière très différente, à la production d'une nouvelle réalité sociale, d'un nouvel espace du nom de Berditschew. Cette ville de Berditschew voit la production d'espaces de la violence, au sein desquels les agressions contre les Juifs deviennent possibles. À l'inverse, les espaces au sein desquels les Juifs pourraient être protégés, recevoir de l'aide ou trouver refuge, sont très fortement restreints par ce processus. C'est dire combien est ici patente la résorption des lieux sûrs, autant au plan physique, qu'au plan des conditions sociales.

Ce qui peut paraître, vu de loin, comme une pièce parfaitement orchestrée, se révèle être, à force de détails, le produit d'un processus d'appropriation et d'attribution. Ce sont tous les acteurs concernés qui produisent, de manière subjective et située, une réalité sociale. En un laps de temps particulièrement court, on assiste à la production d'un espace dans lequel il devient possible d'escorter des milliers de personnes en plein jour à travers la ville et de les assassiner dans un champ sous les yeux de tous. Après le 15 septembre 1941, la ville qui un jour a porté le nom de Berditchev appartient pour toujours au passé. Les contemporains ignorent encore pourtant que la nouvelle ville allemande de Berditschew n'aura qu'une existence éphémère. 
Avant et après le 15 septembre 1941, d'autres exécutions de masse, avec à chaque fois plusieurs centaines de mort, ont lieu - à chaque fois, les victimes sont des Juifs. Les fosses communes sont dispersées autour de la ville. Aujourd'hui, certains de ces lieux sont marqués par des stèles commémoratives mentionnant les crimes commis. Mais étant donné que les plaques proviennent en grande majorité de la période soviétique, les épitaphes ne sont pas en mémoire des Juifs assassinés, mais mentionnent l'assassinat de citoyens soviétiques. En partie cachés, les mémoriaux sont situés en bordure des champs et sur le bas-côté des routes. Les lieux véritables des exécutions de masse, aujourd'hui recouverts de champs et prairies, sont labourés ou laissés en jachère.

\section{Bibliographie}

Altshuler, M. (1993), “Escape and Evacuation of Soviet Jews at the Time of the Nazi Invasion. Policies and Realities", in DobroszYCKI, L., Gurock, J.S. (Eds.). Holocaust in the Soviet Union, New York/London : M.E. Sharpe, pp.77104.

BABerowski, J., MetZler, G. (Eds.) (2012). Gewalträume. Soziale Ordnungen im Ausnahmezustand, Frankfurt am Main : Campus.

Christ, M. (2013). “Codierung von Gewalt”, in Christ, M., GudEHus, G. (Eds.). Gewalt. Ein interdisziplinäres Handbuch, Stuttgart : Metzler, pp.190-196.

Christ, M. (2012). Die Dynamik des Tötens. Die Ermordung der Juden in Berditschew. Ukraine 1941-1944, Frankfurt am Main : Fischer.

EPELFELD, N. (2004). “Möge mein Gedächtnis das Vergessen verhindern... ”, in ZABARKO, B. (Ed.). "Nur wir haben überlebt”. Holocaust in der Ukraine. Zeugnisse und Dokumente, Berlin : Dittrich, pp.110-129.

Löw, M. (2001). Raumsoziologie, Frankfurt am Main : Suhrkamp.

MAURER, M. (2008). Kulturgeschichte. Köln : Böhlau.

SCHLÖGEL, K. (2003). Im Raume lesen wir die Zeit : über Zivilisationsgeschichte und Geopolitik, München : Hanser.

\section{Archives}

USHMM 1996 A 0269 Reel 8 1188-2-421, Gebietskommissar Berditschew, Straßennamen vom 26. April 1942.

Volksbund Deutsche Kriegsgräberfürsorge GN 1.2. Lr, Soldatenfriedhof Berditschew vom 26. Januar 1993. 


\section{Interviews}

Interview de Mariia Jefimowna Beizerman, 14 février 1997, Berditschew / Ukraine, USC Shoah Foundation Institute for Visual History and Education, Interviewcode 27766.

Interview de Mikhail Aronowitsch Vanshelboim, 2 février 1997, Shitomir / Ukraine, USC Shoah Foundation Institute for Visual History and Education, Interviewcode 27225. 